\title{
Physics-based and data-driven hybrid modeling: when data enrich models and models render data smarter
}

\author{
Francisco Chinesta $^{1}$, Elias Cueto ${ }^{2}$ and Jean Louis Duval ${ }^{3}$ \\ ${ }^{1}$ PIMM Lab \& ESI Chair, \\ Arts et Métiers Institute of Technology \\ 151 Boulevard de l'Hôpital, 75013 Paris, France \\ e-mail: Francisco.Chinesta@ensam.eu \\ ${ }^{2}$ I3A, University of Zaragoza \\ Edificio Betancourt. María de Luna, s.n. \\ 50018 Zaragoza, Spain \\ Email: ecueto@unizar.es \\ ${ }^{3}$ ESI Group \\ 3Bis Rue Saarinen, 94528 Rungis, France \\ Email: Jean-Louis.Duval@esi-group.com
}

\begin{abstract}
This presentation aims at revisiting the state of the art and the most recent advances on six domains of data-driven engineering, in particular: (i) visualization of multidimensional data; (ii) classification and clustering, supervised and unsupervised, where it is assumed that members of the same cluster have similar behaviors; (iii) model extraction, that is, discovering the quantitative relationship between inputs (actions) and outputs (reactions), with emphasis in physics-aware data-driven modelling. These three applicative domains have acquired some maturity and proved their abilities in different domains from numerous success-stories. When addressing knowledge extraction, item (iv), as well as the need of explaining for certifying, item (v), advances are much limited and both items need for major progresses, as the one enabling discarding useless parameters, or discovering latent variables whose consideration becomes compulsory for explaining experimental findings, or combining parameters that act in a combined manner, like the velocity, viscosity and density that in fluid mechanics act combined into the so-called Reynolds number. Discovering equations is a very timely topic because it finally enables transforming data into knowledge. Finally, (vi), all the just referred elements are subtlety integrated into the so-called twins, a sort of avatar that allows defining powerful DDDAS (dynamic data-driven application systems), a compulsory protagonist of the new engineering [1].
\end{abstract}

We will emphasize the fact that in the same way that data enrich state-of-the-art physicsbased models, the last contribute to transform big-data needs into a smarter useful-data paradigm, with important consequences in engineering.

\section{REFERENCES}

[1] F. Chinesta, E. Cueto, E. Abisset-Chavanne, J.L. Duval, F. El Khaldi. Virtual, Digital and Hybrid Twins: A New Paradigm in Data-Based Engineering and Engineered Data, Archives of Computational Methods in Engineering, 27, 105-134, 2020. 